PROCEEDINGS OF THE

AMERICAN MATHEMATICAL SOCIETY

Volume 130 , Number 11, Pages 3231-3236

S 0002-9939(02)06494-8

Article electronically published on May 22, 2002

\title{
A QUESTION OF EREMENKO AND LYUBICH CONCERNING COMPLETELY INVARIANT DOMAINS AND INDIRECT SINGULARITIES
}

\author{
WALTER BERGWEILER
}

(Communicated by Juha M. Heinonen)

\begin{abstract}
We give an example of an entire function with a completely invariant Fatou component which has an indirect singularity not contained in this Fatou component. The question of whether such a function exists has been raised by Eremenko and Lyubich.
\end{abstract}

\section{INTRODUCTION AND RESUlT}

Let $f$ be an entire function and $a \in \mathbb{C}$. Then $a$ is called a critical value of $f$ if there exists $z_{0} \in \mathbb{C}$ with $f\left(z_{0}\right)=a$ and $f^{\prime}\left(z_{0}\right)=0$, and $a$ is called an asymptotic value if there exists a curve $\gamma:[0, \infty) \rightarrow \mathbb{C}$ such that $\gamma(t) \rightarrow \infty$ and $f(\gamma(t)) \rightarrow a$ as $t \rightarrow \infty$. The critical and asymptotic values of $f$ form the set $\operatorname{sing}\left(f^{-1}\right)$ of singularities of the inverse function of $f$. The critical values are also called algebraic singularities and the asymptotic values are also called transcendental singularities. The set $\operatorname{sing}\left(f^{-1}\right)$ plays an important role in complex dynamics.

Let $a$ be an asymptotic value and let $\gamma$ be as above. For $r>0$ let $D(a, r):=$ $\{z \in \mathbb{C}:|z-a|<r\}$. By $\Omega(r)$ we denote the component of $f^{-1}(D(a, r))$ which contains the "tail" of $\gamma$; that is, $\Omega(r)$ is the (unique) component of $f^{-1}(D(a, r))$ for which there exists $t_{r} \geq 0$ such that $\gamma\left(\left[t_{r}, \infty\right)\right) \subset \Omega(r)$. We say that $a$ is a direct singularity if there exists $r>0$ such that $f(z) \neq a$ for all $z \in \Omega(r)$. Otherwise $a$ is called an indirect singularity; see [4, §XI.1] for this classification of singularities.

Note that the question of whether $a$ is direct or indirect actually depends not only on $a$ but also on $\gamma$. A more precise terminology would be to say that $a$ is (in)direct with respect to $\gamma$. For example, the function $\exp \left(-z^{2}\right) / \Gamma(z)$ tends to 0 along both the positive and negative real axes, and 0 is direct with respect to the positive real axis and indirect with respect to the negative real axis.

The Fatou set $F(f)$ of $f$ is defined to be the set of all points where the family $\left\{f^{n}\right\}_{n \in \mathbb{N}}$ of iterates of $f$ is normal. It is well known that the Fatou set is completely invariant; that is, $f^{-1}(F(f))=F(f)$. Baker [1] proved that the Fatou set of an entire transcendental function contains at most one completely invariant component. He 2, p. 278] raised the question of whether for such a component $U$ we necessarily have $U=F(f)$. This was proved by Eremenko and Lyubich [3, Theorem 6] in the case that $\operatorname{sing}\left(f^{-1}\right)$ is finite. They deduced this from a general result (see [3.

Received by the editors March 12, 2001.

2000 Mathematics Subject Classification. Primary 37F10; Secondary 30D05, 30D30.

The author was supported by G.I.F., G -643-117.6/1999 and INTAS-99-00089. 
Lemma 11 and Remark 1]) which says that a completely invariant component of $F(f)$ contains all critical values and all direct singularities of $f$. This sharpened an earlier result of Baker [2, Theorem 2]. Eremenko and Lyubich [3, Remark 1] asked whether a completely invariant domain must also contain all indirect singularities. We show that this is not the case.

Example. The function $f$ defined by

$$
f(z)=\frac{12 \pi^{2}}{5 \pi^{2}-48}\left(\frac{\left(\pi^{2}-8\right) z+2 \pi^{2}}{z\left(4 z-\pi^{2}\right)} \cos \sqrt{z}+\frac{2}{z}\right)
$$

is entire and has 0 as an indirect singularity, and $F(f)$ has a completely invariant component $U$ with $0 \in \partial U$.

More specifically, we shall see that 0 is a fixed point of $f$ of multiplier 1 and that $U$ is the parabolic basin associated to 0 . We have $(0, \infty) \subset U$ and $f(x) \rightarrow 0$ as $x \rightarrow \infty, x \in \mathbb{R}$.

By the same method of proof we see that there exists $\alpha_{0}>1$ such that if $1<\alpha<\alpha_{0}$, then $f_{\alpha}(z):=\alpha f(z)$ has an attracting fixed point $x_{\alpha}>0$ whose attracting basin $U_{\alpha}$ is completely invariant and satisfies $0 \in \partial U_{\alpha}$. Clearly, 0 is also an indirect singularity of $f_{\alpha}$.

Computationally we find that $\alpha_{0}=6.348889 \ldots$ and that for $\alpha=2.594992 \ldots$ the fixed point $x_{\alpha}=20.599204 \ldots$ is superattracting.
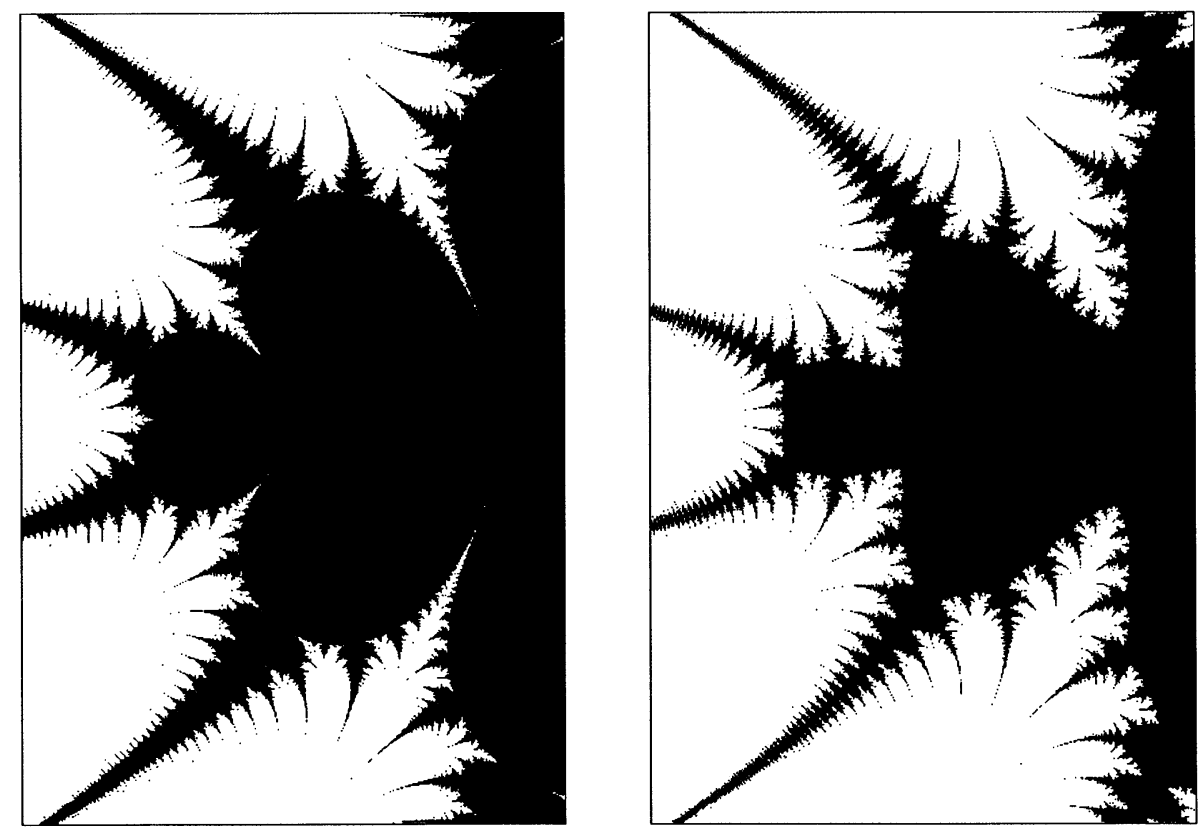

FiguRE 1. The dark sets are the completely invariant components of $f$ (left) and $f_{\alpha}$ with $\alpha=2.594992$ (right). The range shown is $-100 \leq \operatorname{Re} z \leq 300,|\operatorname{Im} z| \leq 300$. 


\section{VERIFICATION OF THE PROPERTIES OF THE EXAMPLE}

Let

$$
g(z):=\frac{z+\frac{2 \pi^{2}}{\pi^{2}-8}}{z-\frac{\pi^{2}}{4}} \cos \sqrt{z}=\left(1+\frac{\pi^{4}}{\left(\pi^{2}-8\right)\left(4 z-\pi^{2}\right)}\right) \cos \sqrt{z}
$$

and

$$
h(z):=\frac{g(z)-g(0)}{z} .
$$

Then $g$ and $h$ are entire and a computation shows that

$$
f(z)=\frac{h(z)}{h^{\prime}(0)} .
$$

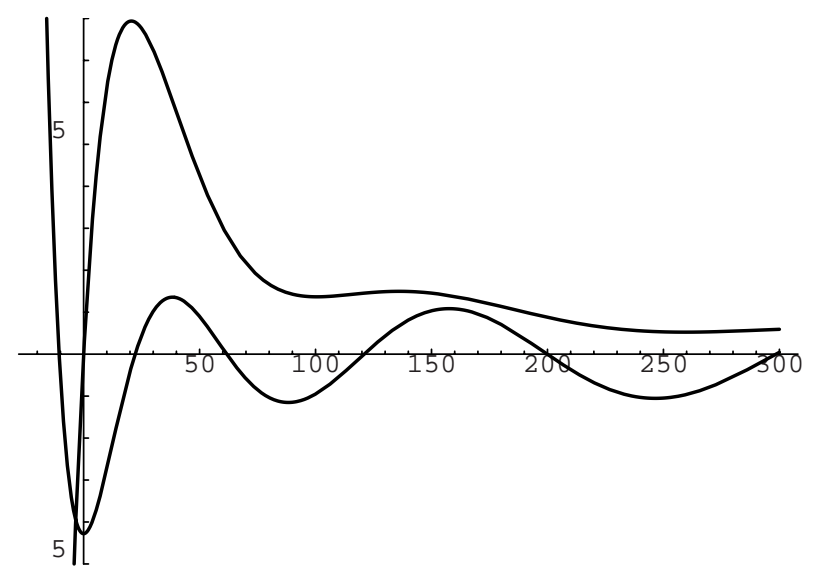

Figure 2. The graphs of $f$ and $g$.

We shall first discuss some properties of the functions $g$ and $h$. We note that $g$ belongs to the Laguerre-Pólya class consisting of all entire functions which can be locally uniformly approximated by polynomials with real zeros and real coefficients. This implies that the only zeros of $g^{\prime}$ are simple, real zeros located in the intervals bounded by the zeros of $g$. Moreover, direct computation shows that $g^{\prime}(0)=0$. To summarize, if we denote the zeros of $g^{\prime}$ by $\xi_{k}$, with $\xi_{1}<\xi_{2}<\xi_{3}<\ldots$, then

(i) $\xi_{1}=0$ and $\xi_{k} \in\left(\left(k-\frac{1}{2}\right)^{2} \pi^{2},\left(k+\frac{1}{2}\right)^{2} \pi^{2}\right)$ for $k \geq 2$.

We see that $g$ has local maxima at $\xi_{2 k}$ and local minima at $\xi_{2 k-1}$ for $k \in \mathbb{N}$. For $x \geq \xi_{2} \geq \frac{9}{4} \pi^{2}$ we have

$$
|g(x)| \leq\left|1+\frac{\pi^{4}}{\left(\pi^{2}-8\right)\left(4 x-\pi^{2}\right)}\right| \leq 1+\frac{\pi^{2}}{8\left(\pi^{2}-8\right)}<\frac{4}{\pi^{2}-8}=-\frac{g(0)}{2} .
$$

This implies that

(ii) $g(x)>g(0)$ for $x \in \mathbb{R} \backslash\{0\}$ and $g(x)<-\frac{1}{2} g(0)$ for $x \in[0, \infty)$.

Now we consider the function $h$. We have

$$
h^{\prime}(z)=M(z)-\frac{8}{\left(\pi^{2}-8\right) z^{2}}+R(z) \cos \sqrt{z}
$$


where

$$
M(z):=-\frac{z+\frac{2 \pi^{2}}{\pi^{2}-8}}{z\left(z-\frac{\pi^{2}}{4}\right)} \cdot \frac{\sin \sqrt{z}}{2 \sqrt{z}}
$$

and

$$
R(z):=-\frac{z^{2}+\frac{4 \pi^{2}}{\pi^{2}-8} z-\frac{\pi^{4}}{2\left(\pi^{2}-8\right)}}{z^{2}\left(z-\frac{\pi^{2}}{4}\right)^{2}}=O\left(\frac{1}{|z|^{2}}\right)
$$

as $z \rightarrow \infty$. A computation shows that $h^{\prime}\left(\pi^{2}\right)>0$ and $(-1)^{k+1} h^{\prime}\left(\left(k+\frac{1}{2}\right)^{2} \pi^{2}\right)>0$ for $k \geq 2$. The intermediate value theorem implies that $h^{\prime}$ has a zero in the interval $\left(\pi^{2},\left(\frac{5 \pi}{2}\right)^{2}\right)$ and in each interval $\left(\left(k+\frac{1}{2}\right)^{2} \pi^{2},\left(k+\frac{3}{2}\right)^{2} \pi^{2}\right)$ for $k \geq 2$. To show that there is only one zero in each such interval and that there are no further zeros of $h^{\prime}$, we consider for $k \in \mathbb{N}$ and $T>0$ the simple closed curve

$$
\Gamma:=\left\{\left(\left(k+\frac{1}{2}\right) \pi+i y\right)^{2}:|y| \leq T\right\} \cup\left\{(x \pm i T)^{2}: 0 \leq x \leq\left(k+\frac{1}{2}\right) \pi\right\} .
$$

If $k$ and $T$ are large enough, then we have

$$
\left|h^{\prime}(z)-M(z)\right|=\left|-\frac{8}{\left(\pi^{2}-8\right) z^{2}}+R(z) \cos \sqrt{z}\right|<|M(z)|
$$

for $z \in \Gamma$. Rouché's theorem implies that the number of zeros of $h^{\prime}$ in the interior of $\Gamma$ equals the number of zeros minus the number of poles of $M$ in the interior of $\Gamma$. Now $M$ has $k+1$ zeros at $-\frac{2 \pi^{2}}{\pi^{2}-8}, \pi^{2}, 4 \pi^{2}, \ldots, k^{2} \pi^{2}$ and two poles at 0 and $\frac{\pi^{2}}{4}$ there. Hence $h^{\prime}$ has $k-1$ zeros in the interior of $\Gamma$, and we conclude that $h^{\prime}$ has no zeros besides the ones obtained from the intermediate value theorem. To summarize, if we denote the zeros of $h^{\prime}$ by $\eta_{k}$, with $\eta_{1}<\eta_{2}<\eta_{3}<\ldots$, then

(iii) $\eta_{1} \in\left(\pi^{2},\left(\frac{5 \pi}{2}\right)^{2}\right)$ and $\eta_{k} \in\left(\left(k+\frac{1}{2}\right)^{2} \pi^{2},\left(k+\frac{3}{2}\right)^{2} \pi^{2}\right)$ for $k \geq 2$.

We now find that $h$ has local maxima at $\eta_{2 k-1}$ and local minima at $\eta_{2 k}$ for $k \in \mathbb{N}$. Using (ii) we find for $x \geq \eta_{3} \geq \frac{49}{4} \pi^{2}$ that

$$
h(x) \leq \frac{-\frac{3}{2} g(0)}{\frac{49}{4} \pi^{2}}=\frac{48}{49 \pi^{2}\left(\pi^{2}-8\right)}<\frac{32}{25 \pi^{2}\left(\pi^{2}-8\right)}=h\left(\frac{25}{4} \pi^{2}\right) \leq h\left(\eta_{1}\right) .
$$

It follows that $h$ attains its maximum on $\mathbb{R}$ at $\eta_{1}$. Combining this with (ii) we see that

(iv) $0<h(x) \leq h\left(\eta_{1}\right)$ for $x \in(0, \infty)$.

Next we observe that $h^{\prime}$ has order $\frac{1}{2}$. This implies that $h^{\prime}$ also belongs to the Laguerre-Pólya class. It follows that the only zeros of $h^{\prime \prime}$ are simple zeros in the intervals $\left(\eta_{k}, \eta_{k+1}\right), k \in \mathbb{N}$. In particular, $h^{\prime \prime}(x) \neq 0$ for $x<\eta_{1}$. Thus

(v) $h^{\prime}$ is decreasing on the interval $\left(-\infty, \eta_{1}\right]$.

Finally we consider the function $f$. Of course, the $\eta_{k}$ are also the zeros of $f^{\prime}$, and since $h^{\prime}(0)>0$ we conclude that (iv) and (v) hold with $h$ replaced by $f$. We have $f(0)=0, f^{\prime}(0)=1$ and $f^{\prime \prime}(0)<0$. Thus 0 is a fixed point of $f$ of multiplier 1 , with one immediate parabolic basin associated to it. We denote this parabolic basin by $U$. From (v) we obtain $0<f^{\prime}(x)<1$ for $x \in\left(0, \eta_{1}\right)$ and $f^{\prime}(x)>1$ for $x<0$. This implies that $f^{n}(x) \rightarrow-\infty$ as $n \rightarrow \infty$ if $x<0$ and that $\left(0, \eta_{1}\right] \subset U$. Using (iv) we see that in fact $(0, \infty) \subset U$. 
It is not difficult to see that $f(x) \rightarrow 0$ as $x \rightarrow \infty, x \in \mathbb{R}$. It follows from the result of Eremenko and Lyubich [3] mentioned in the introduction, or the DenjoyCarleman-Ahlfors theorem [4, §XI.4], or direct computation that 0 is an indirect singularity. The Denjoy-Carleman-Ahlfors theorem also implies that $f$ has no other (finite) asymptotic values. Thus $\operatorname{sing}\left(f^{-1}\right) \subset\left[0, f\left(\eta_{1}\right)\right] \subset[0, \infty)$.

Next we show that the positive real axis is the only curve where $f$ tends to zero and is real and positive. More precisely, we show that there does not exist a curve $\gamma:[0, \infty) \rightarrow \mathbb{C} \backslash[0, \infty)$ with $\gamma(t) \rightarrow \infty$ and $f(\gamma(t)) \rightarrow 0$ as $t \rightarrow \infty$ such that $f(\gamma(t)) \in(0, \infty)$ for all $t$. In fact, suppose that such a curve $\gamma$ exists and let

$$
L:=\left\{(u+i v)^{2}:|u| \geq 1,|v| \leq 4 \log |u|\right\} .
$$

For $z=(u+i v)^{2} \in \mathbb{C} \backslash L$ we have

$$
|\cos \sqrt{z}|=|\cos (u+i v)| \geq \frac{1}{2}\left(e^{|v|}-1\right) \geq \frac{1}{2}\left(u^{4}-1\right)
$$

and

$$
|\cos \sqrt{z}| \geq \frac{1}{2}\left(e^{|v|}-1\right) \geq \frac{1}{48} v^{4} .
$$

Because $|z|^{2}=\left(u^{2}+v^{2}\right)^{2} \leq 4 \max \left\{u^{4}, v^{4}\right\}$ we obtain $|\cos \sqrt{z}| \geq \frac{1}{192}|z|^{2}-\frac{1}{2}$ for $z \in \mathbb{C} \backslash L$ and thus $|f(z)| \rightarrow \infty$ as $z \rightarrow \infty, z \in \mathbb{C} \backslash L$. Hence $\gamma(t) \in L$ for sufficiently large $t$.

For $k \in \mathbb{N}$ we define

$$
\begin{aligned}
L_{k} & :=L \cap\left\{\left(\left(2 k+\frac{1}{2}\right) \pi+i v\right)^{2}: v \in \mathbb{R}\right\} \\
& =\left\{\left(\left(2 k+\frac{1}{2}\right) \pi+i v\right)^{2}:|v| \leq 4 \log \left(\left(2 k+\frac{1}{2}\right) \pi\right)\right\} .
\end{aligned}
$$

Then $\gamma$ intersects $L_{k}$ for sufficiently large $k$, say $\gamma\left(t_{k}\right)=\left(\left(2 k+\frac{1}{2}\right) \pi+i v_{k}\right)^{2} \in L_{k}$.

We note that for $z=(u+i v)^{2} \in L$ we have $|v|=O(\log |u|)=o(|u|)$ and hence

$$
|z|=u^{2}+v^{2}=u^{2}\left(1+O\left(\left(\frac{\log |u|}{|u|}\right)^{2}\right)\right)
$$

as $z \rightarrow \infty$. We thus have

$$
\Re\left(\frac{1}{z}\right)=\frac{u^{2}-v^{2}}{|z|^{2}}=\frac{1}{u^{2}}\left(1+O\left(\left(\frac{\log |u|}{|u|}\right)^{2}\right)\right)
$$

and

$$
\Im\left(\frac{1}{z}\right)=\frac{-2 u v}{|z|^{2}}=-\frac{2 v}{u^{3}}\left(1+O\left(\left(\frac{\log |u|}{|u|}\right)^{2}\right)\right) .
$$

Now

$$
h(z)=\left(\frac{1}{z}+O\left(\frac{1}{z^{2}}\right)\right) \cos \sqrt{z}-\frac{g(0)}{z}
$$


as $z \rightarrow \infty$. With $z_{k}:=\gamma\left(t_{k}\right)=\left(\left(2 k+\frac{1}{2}\right) \pi+i v_{k}\right)^{2} \in L_{k}$ we have $\cos \sqrt{z_{k}}=$ $\cos \left(\frac{\pi}{2}+i v_{k}\right)=-i \sinh v_{k}$ and thus we obtain

$$
\begin{aligned}
\Im h\left(z_{k}\right)= & -\sinh v_{k} \frac{1}{\left(2 k+\frac{1}{2}\right)^{2} \pi^{2}}\left(1+O\left(\left(\frac{\log k}{k}\right)^{2}\right)\right) \\
& +v_{k} \frac{2 g(0)}{\left(2 k+\frac{1}{2}\right)^{3} \pi^{3}}\left(1+O\left(\left(\frac{\log k}{k}\right)^{2}\right)\right) .
\end{aligned}
$$

It follows that $\Im f\left(z_{k}\right)=\left(\Im h\left(z_{k}\right)\right) / h^{\prime}(0) \neq 0$ for sufficiently large $k$, a contradiction. Hence a curve $\gamma$ as above does not exist.

We now show that $U$ is completely invariant. We assume that this is not the case. Then $f^{-1}(U)$ has a component $V \neq U$. Since the zeros of $f^{\prime}$ are real and positive, and since 0 is the only asymptotic value, $f$ maps $V$ biholomorphically onto $U$. Let $\varphi$ be the branch of $f^{-1}$ which maps $U$ to $V$. Then $\varphi(x) \nrightarrow \infty$ as $x \rightarrow 0, x>0$, since otherwise $\varphi$ would define a curve tending to infinity where $f$ is real and positive and tends to 0 . Thus $\varphi$ accumulates at some finite point; that is, for $A:=\bigcap_{\varepsilon>0} \overline{\varphi((0, \varepsilon])}$ we have $A \cap \mathbb{C} \neq \emptyset$. Since $A \cap \mathbb{C}$ can consist only of zeros of $f$ and since $A$ is connected, we in fact have $A=\left\{z_{0}\right\}$ for some zero $z_{0}$ of $f$. Thus $\varphi(t) \rightarrow z_{0}$ as $t \rightarrow 0$. Since 0 is not a critical value of $f$ we have $f^{\prime}\left(z_{0}\right) \neq 0$ and thus $\varphi$ has an analytic continuation to a neighborhood of 0 . Since $\operatorname{sing}\left(f^{-1}\right) \subset[0, \infty) \subset U \cup\{0\}$ we see that $\varphi$ can also be continued analytically to any point of $\mathbb{C} \backslash U$. This implies that $\varphi$ extends to an entire function. Since $\varphi$ is also univalent, we deduce that $\varphi$ and hence $f$ are linear, a contradiction.

\section{ACKNOWLEDGMENT}

I thank the referee for helpful comments, in particular for a suggestion on how to prove that $U$ is completely invariant. My original proof used a different argument.

\section{REFERENCES}

[1] I. N. Baker, Completely invariant domains of entire functions, in Mathematical Essays Dedicated to A. J. Macintyre, edited by H. Shankar, Ohio University Press, Athens, Ohio, 1970, 33-35. MR 42:6227

[2] The domains of normality of an entire function, Ann. Acad. Sci. Fenn. (Ser. A, I. Math.) 1 (1975), 277-283. MR 53:5867]

[3] A. E. Eremenko and M. Yu. Lyubich, Dynamical properties of some classes of entire functions, Ann. Inst. Fourier 42 (1992), 989-1020. MR 93k:30034

[4] R. Nevanlinna, Analytic Functions, Springer, Berlin, Heidelberg, New York, 1970. MR 43:5003

Mathematisches Seminar, Christian-Albrechts-Universität Zu Kiel, Ludewig-MeynStr. 4, D-24098 Kiel, Germany

E-mail address: bergweiler@math.uni-kiel.de 